erklärte Cornberg. Bei einigen Patienten komme es unter PEGIFN zu einem Verlust des HBsAg, was eine serologische und klinische Ausheilung bedeutet. Um die Heilungsrate zu steigern werden zurzeit Studien mit Kombinationen von NA plus PEGIFN durchgeführt oder IFN in einer NA-Pause gegeben. Doch bisher reiche die Evidenz nicht aus, um solche Therapiestrategien empfehlen zu können. Auch seien innovative Medikamente zur Stimulation des Immunsystems und auch Inhibitoren, die Replikationsschritte des HBV hemmen, in der klinischen Entwicklung. Das Therapieziel bleibe „HBV-Cure“ oder zumindest eine funktionale Heilung.

\section{Hochreplikative HBV-Infektion}

Ein seit vielen Jahren intensiv diskutiertes Thema ist, ob alle HBV-Infizierten sofort behandelt werden sollten. Nicht selten findet sich vor allem bei jungen Patienten eine sehr hohe HBVReplikation ohne entzündliche Aktivität, also ohne Erhöhung der Transaminasen. Eine solche Konstellation wurde, so Cornberg, früher als immuntolerante Phase einer chronischen HBVInfektion bezeichnet. In der aktualisierten Leitlinie wird dies jetzt als hochreplikative HBV-Infektion, aber nicht mehr als $\mathrm{He}$ - patitis B bezeichnet. „Wenn diese Patienten jünger als 30 Jahre sind und keine weiteren Risikofaktoren für ein HCC haben, besteht keine Behandlungsindikation.“

\section{Vertikale Transmission verhindern}

Bei Schwangeren muss das Risiko einer vertikalen Transmission auf das Neugeborene bedacht werden. Studien haben gezeigt, dass dieses Risiko durch Tenofovir deutlich gesenkt werden kann. In der neuen Leitlinie wird deshalb empfohlen, Schwangere mit einer HBV-DNA > 200.000 IU/ml zu behandeln. Zirka $50 \%$ aller HBV-Infizierten sind inaktive Virusträger, das heißt die HBV-DNA ist mit einem Titer $<2.000 \mathrm{IU} / \mathrm{ml}$ niedrig und die Transaminasen sind nicht erhöht. „Doch dies kann sich schnell ändern, nämlich wenn diese Patienten mit Immunsuppressiva behandelt werden“, so Cornberg. Deshalb sollten alle Patienten, bevor mit einer solchen Therapie begonnen wird, auf HBsAg und anti-HBc getestet und bei entsprechender Konstellation auch prophylaktisch behandelt werden. Dr. med. PeterStiefelhagen

Neue Leitlinien der DGVS. Jahrestagung der DGVS \& 11. Herbsttagung der DGAV, 14.9.2107 in Dresden, Viszeralmedizin 2017, 14.9.2107 in Dresden

\title{
NAFDL und Typ-2-Diabetes: Henne oder Ei?
}

\section{Schon länger im Fokus der Hepatologen stehen NAFLD (non alcoholic fatty liver disease) und NASH (non alcoholic steatohepatitis). Aus gutem Grund: Im- merhin ist die NAFLD die häufigste Lebererkrankung in der westlichen Welt.}

Die NAFLD hat eine globalen Prävalenz von $25 \%$, in Populationen mit den metabolischen Risikofaktoren Übergewicht, Insulinresistenz und Typ-2-Diabetes bei bis zu 70\%. Dabei ist noch immer unklar, was zuerst da ist, betonte Professor Jörg Bojunga, Frankfurt: Ist die NAFLD Folge von Diabetes und Insulinresistenz oder treibt sie die Entwicklung der Insulinresistenz voran? Aus seiner Sicht sollte mehr Augenmerk auf die NAFLD als Verursacher der Gesamtproblematik gelegt werden.

\section{Problem Progression}

Die hohe Prävalenz der NAFDL ist aber nicht das alleinige Problem. Es ist auch die Progression, wie Dr. Anita Pathil-Warth,

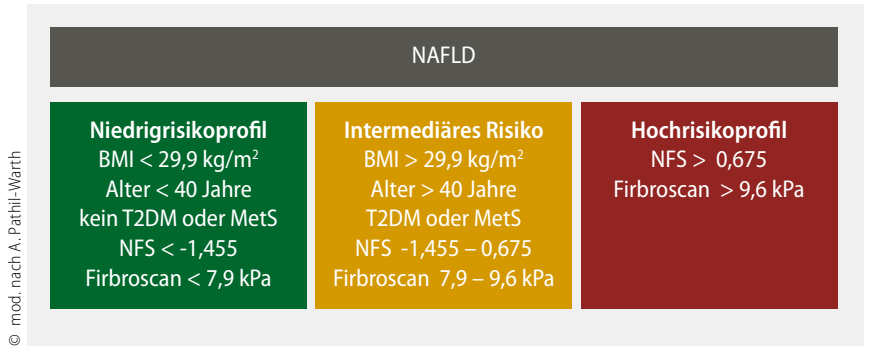

1

Risikostratifizierung NAFLD
Heidelberg, deutlich machte. Liegt eine NAFDL vor, steigt das Risiko für Zirrhose und Fibrose, aber auch für ein hepatozelluläres Karzinom (HCC). „NAFLD ist nach den viralen Hepatitiden der häufigste Risikofaktor für ein HCC“, so Pathil-Warth, „davon ein relevanter Anteil in der nicht zirrhotischen Leber“. Insgesamt ist bei einer NAFDL die leberassoziierte und die Gesamtmortalität erhöht, darunter auch das Risiko für kardiovaskuläre Erkrankungen und extrahepatische Malignome. Sie forderte deshalb ein Screening zur Stratifizierung und Identifikation von Risikopatienten. Wichtigster Prädiktor für einen negativen Verlauf ist das Fibrosestadium. Die höhergradige Fibrose (F3 und F4) ist der wichtigste singuläre Parameter mit der höchsten prognostischen Relevanz. Klinische Prädiktoren für eine höhergradige Fibrose sind das Alter des Patienten, eine Adipositas sowie ein Typ-2-Diabetes. Zur Bestimmung des Fibrosegrads stehen der NAFLD-Fibrosis-Score (NFS) und der Fibroscan zur Verfügung. Eine Leberbiospie hält sie für das Screening nicht vertretbar, sie kann aber im weiteren Verlauf zum Zuge kommen. ( $\triangleright$ Abb.1)

An der Therapie von NAFDL und NASH wird mit großem Engagement und Feuereifer geforscht. Die Wissenschaftler setzen auf Substanzen, die an nukleären Rezeptoren andocken, wie etwa FXR-AGonisten, PPAR-AGonisten oder auch ChemokinRezeptorantagonisten oder auch FGF-10-Agonisten. Bis zur Zulassung hat es zumindest bislang noch kein Wirkstoff geschafft. Die Strategien beschränken sich deshalb derzeit darauf, die Stoffwechselsituation zu optimieren, durch Gewichtsreduktion und Verbesserung von Insulinresistenz und Dysipidämie.

Dr. Beate Fessler

Nicht alkoholische Fettlebererkrankung: Screening-Strategien zur Erkennung der NAFLD-Risikopatienten. Viszeralmedizin 2017, 15.9.2017 in Dresden 